\title{
The Use of Liquid Crystal Thermography in Selected Technical and Medical Applications-Recent Development
}

\author{
Jan Stasiek $^{1}$, Marcin Jewartowski ${ }^{1}$, Tomasz Aleksander Kowalewski ${ }^{2}$ \\ ${ }^{1}$ Faculty of Mechanical Engineering, Gdansk University of Technology (GUT), Gdansk, Poland; ${ }^{2}$ Institute of Fundamental Technol- \\ ogical Research Polish Academy of Sciences (IPPT PAN), Warszawa, Poland. \\ Email: jstasiek@pg.gda.pl \\ Received November $26^{\text {th }}, 2013$; revised December $26^{\text {th }}$, 2013; accepted January $3^{\text {rd }}, 2014$ \\ Copyright (C) 2014 Jan Stasiek et al. This is an open access article distributed under the Creative Commons Attribution License, \\ which permits unrestricted use, distribution, and reproduction in any medium, provided the original work is properly cited. In accor- \\ dance of the Creative Commons Attribution License all Copyrights (C) 2014 are reserved for SCIRP and the owner of the intellectual \\ property Jan Stasiek et al. All Copyright (C) 2014 are guarded by law and by SCIRP as a guardian.
}

\section{ABSTRACT}

Thermochromic liquid crystals (TLC) and true-colour digital image processing have been successfully used in non-intrusive technical, industrial and biomedical studies and applications. Thin coatings of TLC at surfaces are utilized to obtain detailed temperature distributions and heat transfer rates for steady or transient processes. Liquid crystals also can be used to make the temperature and velocity fields in liquids visible by the simple expedient of directly mixing the liquid crystal material into the liquid (water, glycerol, glycol, and silicone oils) in very small quantities to use as thermal and hydrodynamic tracers. In biomedical situations, e.g., skin diseases, breast cancer, blood circulation and other medical application, TLC and image processing are successfully used as an additional non-invasive diagnostic method especially useful for screening large groups of potential patients. The history of this technique is reviewed, principal methods and tools are described and some examples are presented. Also steady-state and transient liquid crystal thermography (LCT) is used to measure local heat transfer on a plate equipped with transverse vortex generators. Automated evaluation allows determining the heat transfer coefficient without arbitrary influence of human interpretation.

\section{KEYWORDS}

\section{Liquid Crystal Thermography; Steady-State and Transient Method; Vortex Generators; Biomedicine}

\section{Introduction}

Liquid crystals have emerged as reliable temperature and colour sensors for heat transfer, fluid flow and biomedical research, and have been applied in a number of situations to visualize the temperature distribution under complex flow fields and applications such as medical imaging, machine vision, astronomy, satellite reconnaissance, and even desktop publishing. During this same period, there has been an increasing use of desktop computers in scientific and industrial applications, first to facilitate conventional experiments and then to expand the range of possible experiments. These two new tools have come together during the past ten years to produce a powerful new experimental technique: true-colour digital processing of liquid crystal images to yield full-field measurements of temperature and heat transfer coefficient distribu- tions, as a judgment of food quality, and for electronic components and colour photographs, among other applications.

Also in the last decade, liquid crystals have been extensively applied to the qualitative visualisation of entire steady state, or transient temperature fields on solid surfaces. Since quantifying colour is a difficult and somewhat ambiguous task, application of thermochromic liquid crystals initially was largely qualitative. Application of the colour films or interference filters was tedious and inaccurate. The first application of true-colour digital image processing gave impact to qualitative and fast temperature measurements. Rapid development of the hardware and software of computerised image processing techniques has made it now possible to set up inexpensive systems capable of real-time transient full field temperature measurements using TLCs. 
Within this objective, a new experimental technique has been developed and applied to the study of heat and mass transfer, and for biomedical diagnosis.

\section{Monochrome or Colour: The HSI Advantage}

There are several different ways to specify colour. To producers and users of colour monitors, video cameras, and computer graphics, colour is often thought of as a combination of the primary colours-red, green, and blue (RGB). These additive primaries can be mixed in any combination to produce millions of colours in the visible spectrum. Another traditional colour specification method, popular in the publishing industry for mixing inks, is based on combining the subtractive primaries-cyan, yellow, magenta, and black (CYMB). Television broadcast represents colour with yet another method: RGB signals are encoded into luminance $(\mathrm{Y})$ and chrominance (I and Q) signals to facilitate broadcasting.

Colour image processing can be much less complicated and quicker to execute if colour images captured from RGB video sources can be digitally converted from RGB data to hue-saturation-intensity (HSI) data [1,2].

In addition to facilitating straightforward computer processing, thinking about and specifying colour in terms of HSI values may closely approximate the way humans perceive and interpret colour. Hue, for example, is a colour attribute that describes a pure colour, such as "pure red", "pure yellow", "pure green”, "pure blue”, "pure purple", or some intermediate between these. Hue is what we are typically referring to when we use the term "colour". Saturation is another colour attribute that describes the degree to which a pure colour is diluted with white. A highly saturated colour has a low white content. Intensity is a colour-neutral attribute that describes the relative brightness or darkness. The intensity of a colour image corresponds to the grey-level (black-and-white) version of the image.

To reduce the subjectivity of specifying colour, the Commission International de l'Eclairage (CIE) conducted an experiment in the late 1920's in which a number of individuals were asked to match a test light colour by combining red, green, and blue lights. The experiment's results served as the basis for a set of standardized colour matching functions which showed how much of each primary colour is required to produce a desired colour.

The RGB primaries were used to produce an equilateral triangular colour map. The triangle, with the three primary colours as its vertices, maps out the largest range of reproducible colours in the visual spectrum. Further work extended this to the development of a standardized colour chart, which quantified the perception of colours by assigning numbers to them. The chart, still in wide use, is the 1931 chromaticity diagram. A variation of this diagram, produced in 1976, is called the Uniform Colour Space. Although its creation is based mainly on combinations of the visual-spectrum RGB primary colours, the 1976 chromaticity diagram provides an excellent plot of colour in terms of hue and saturation (Figure 1). Pure

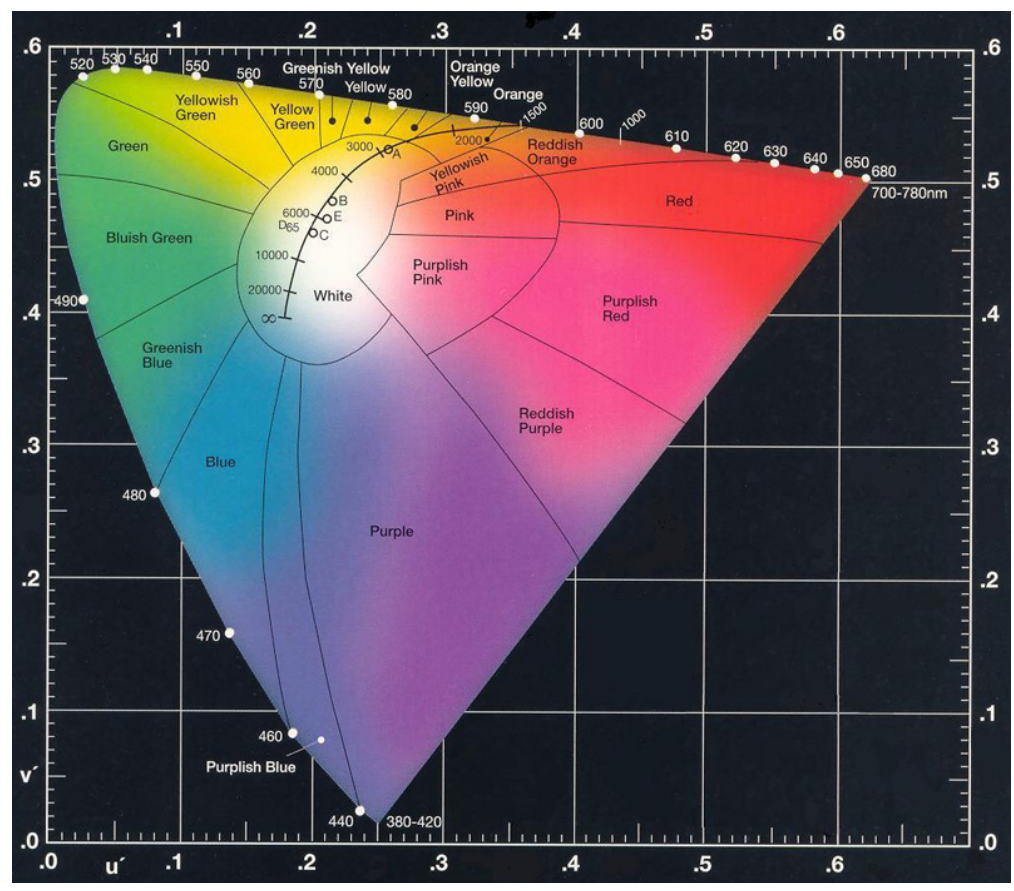

Figure 1. The map of the 1976 chromaticity diagram is similar in nature to the triangle colour map. At the end points of the chart, the colour primaries (as defined by the CIE in 1976) are shown. 
hues, for example, can he selected by moving equidistantly around the perimeter of the 1976 chromaticity diagram. Each point on the diagram's perimeter represents a pure hue. That is, each of these hues is $100 \%$ saturated. The saturation of a chosen hue can be decreased by moving away from the pure-hues perimeter of the diagram toward its centre to the point where red, green, and blue mix equally into while. Each step toward the diagram's centre represents a decrease in saturation.

To perform image processing on a desktop, systems developers can choose from a variety of frame grabbers, array processors, and display controllers. Most of today's monochrome frame grabbers are capable of interfacing to RS-170 and/or CCIR-compatible cameras. Such frame grabbers generally digitize the monochrome analog signal to a precision of 8 bits. This results in a $512 \times 512$ interlaced image with each pixel having 256 grey levels. Because the intensity component of the analog RS-170 signal is digitized, no colour information is present. Fortunately, in many applications the intensity values in the image are all that is required to perform some decisionmaking processes. When certain intensity levels within the image need to be highlighted; however, it is useful to falsely colour, or pseudo-colour, the digitized monochrome image. For example, PC-based frame grabbers and display controllers use look-up tables (LUTs) and digital-to-analog converters (DACs) for this purpose.

Unlike simple monochrome frame grabbers, the display section of such boards requires the use of three DACs to drive the RGB (red, green, blue colour mode) colour display. Also, in monochrome image processing, digitizing colour requires greater hardware complexity. In true colour imaging, images are generally digitized to 8 bits per colour component (RGB). Storing colour image as 8 bits per RGB plane results in a 24-bit image. This representation is known as an additive model because colour pixel intensity values within each RGB plan individually drive the three electron guns of the colour monitor and sum to produce the final colour. To represent the RGB colour model graphically, a colour cube is often used (Figure 2). Here the main axes of the cube are the red, green, and blue components, with white to black on a diagonal connecting the corners of the cube. For any specific application, especially in computer graphics, the RGB model is the most useful. However, because the RGB model is not based on perceptual information, it is less useful in image-processing applications where colour image information needs to be extended [1,2].

Mathematically, it is relatively easy to change a colour or an entire colour image from the RGB colour space to the HSI colour space. If the RGB coordinates in Figure 3 are thought of as being produced by an RGB camera, then the intensity of any colour at that point will be given by

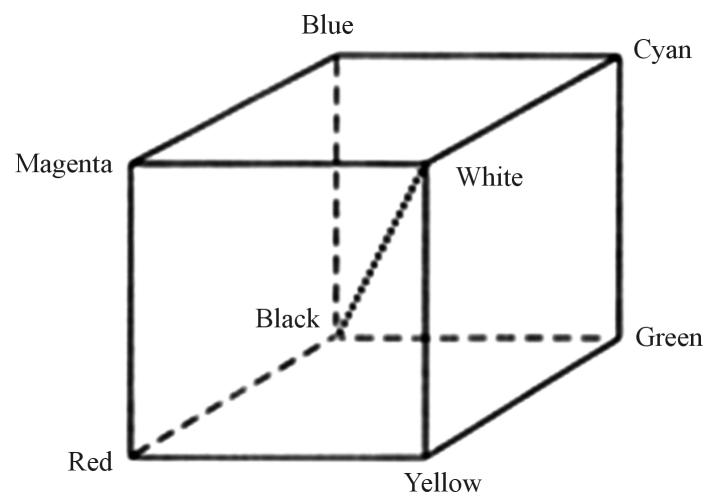

Figure 2. RGB colour cube (greys are on the dotted main diagonal).

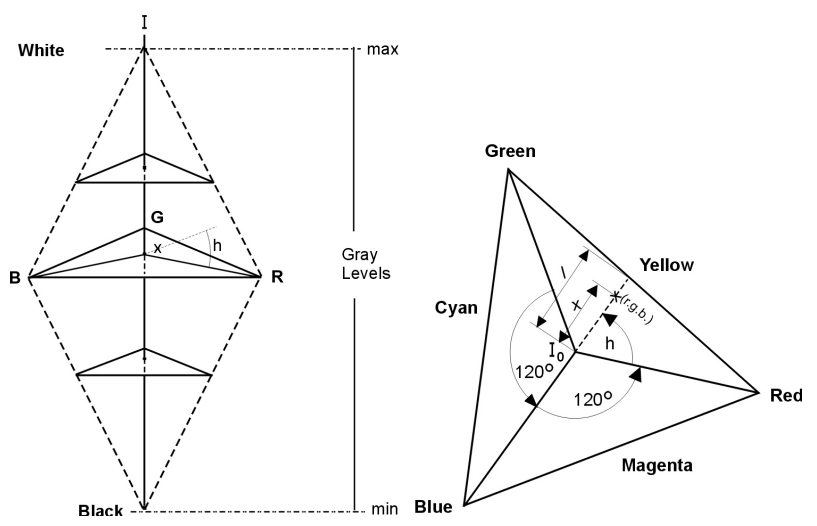

Figure 3. HSI triangle model.

$$
I=(R+B+G) / 3
$$

Further, the hue of the colour can be represented as the angle resulting from a vector rotating about the white point ( $R=G=B$ point). A hue angle of zero degrees corresponds to any point on the line drawn from the white point at the triangle's centre to the red vertex; thus, hue can be given by:

$$
\begin{aligned}
H= & (1 / 360) \\
& \times(90-\operatorname{Arctan}(F / \sqrt{3}+\{0, G>B ; 180, G<B\}))
\end{aligned}
$$

where

$$
I=(2 R-B-G) /(G-B)
$$

Saturation can be obtained by:

$$
S=1-[\min (R, G, B)] / I
$$

In Equation (4), the lowest value of either $R / I, G / I$, or $B / I$ is subtracted from 1 to give the saturation. Thus, for a colour with zero saturation (white), $R / I=G / I=B / I=1$. Substituting into Equation (4) gives that value of saturation as 0 .

Certainly, HSI space allows programmers and users alike to think more naturally in terms of colour. A whiter 
shade of pale green can be thought of as a less saturated red. Finally, different colours can be regarded as different hues. From graphic design and electronic prepress to medical and scientific imaging to medicine vision, intuitive colour specification can add another dimension to image-processing systems.

Digital computers have been brought to bear on the problem of processing natural-quality imagery, and with surprising success. Optical processes, communication systems, and automatic image detection are three areas in which computer techniques are being applied. Other area of research is image restoration and enhancement.

The true colour image processing systems, containing appropriate hardware and software, are currently commercially available from different manufacturers. A brief description of one of the systems is given by Stasiek et al. [3-5].

\section{Liquid Crystal Thermography}

Liquid crystals are temperature indicators that modify incident white light and display colour whose wavelength is proportional to temperature. They can be painted on a surface or suspended in the fluid and used to make visible the distribution of temperature. Normally clear, or slightly milky in appearance, liquid crystals change in appearance over a narrow range of temperature called the "colour-play interval" (the temperature interval between first red and last blue reflection), centred around the nominal "event temperature". It can be selected for bands of about $0.5^{\circ} \mathrm{C}$ to $20^{\circ} \mathrm{C}$, and event temperature of $-30^{\circ} \mathrm{C}$ to above $120^{\circ} \mathrm{C}$. These colour changes are repeatable and reversible as long as the TLC's are not physically or chemically damaged. The response time of TLC's equals about $10 \mathrm{~ms}$. The displayed colour is red at the low temperature margin of the colour-play interval and blue at the high end (Figure 4). Within the colour-play interval, the colours change smoothly from red to blue as a function of temperature. Pure liquid crystal materials are thick, viscous liquids, greasy and difficult to deal with under most heat transfer laboratory conditions [6,7]. The TLC material is also sensitive to mechanical stress. A micro-encapsulation process which encloses small portions of liquid crystal material in polymeric material was introduced to solve problems with the stress sensitivity as well as with the chemical deterioration.

For surface temperature measurements application of the un-encapsulated material (unsealed liquids) to a clear plastic sheet and sealing it with a black backing coat to form a pre-packaged assembly is used. Commercially available temperature indicating devices using TLC, contain a thin film of the liquid crystal sandwiched between a transparent polyester sheet and a black absorbing background, however in the current experiment the liquid crystals are deposited on the black painted sheet. For

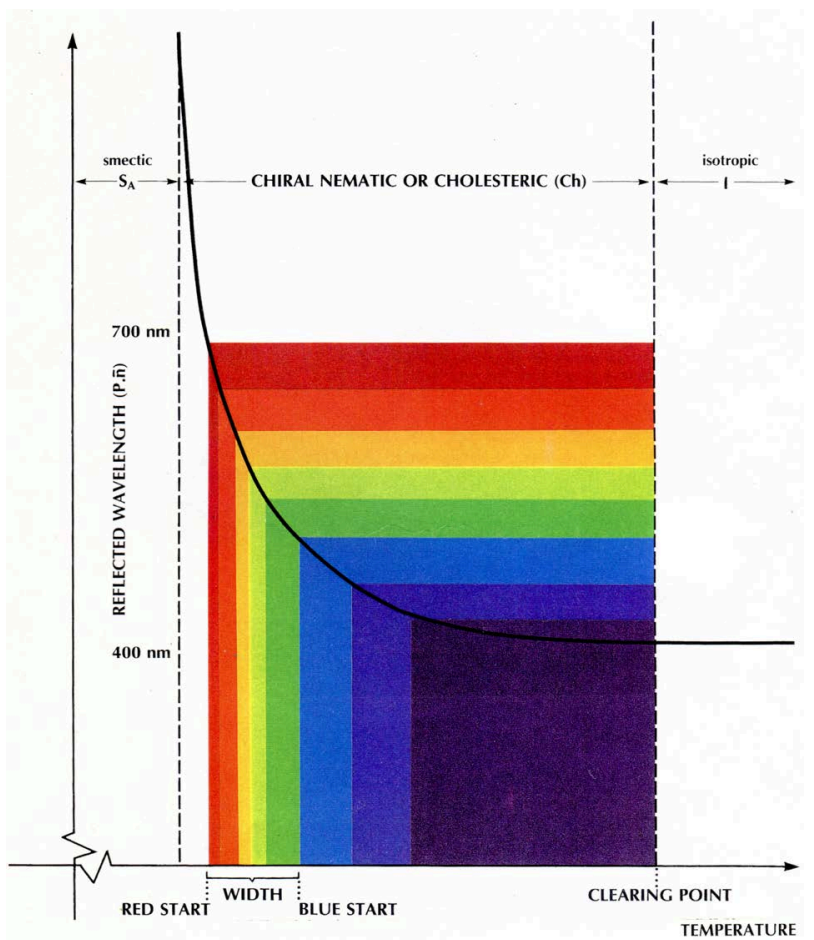

Figure 4. Typical reflected wavelength (colour) temperature response of a TLC mixture.

flow analysis the suspension of thermochromic liquid crystals can be used to make visible the temperature and velocity fields in liquids. By dispersing the liquid crystal material into the liquid they become not only classical tracers used for flow visualisation but simultaneously small thermometers monitoring local fluid temperature [3,8-11]. A collimated source of white light must be used to illuminate a selected cross-section of the flow (light sheet technique) and colour images are acquired in a perpendicular direction.

In the following examples the un-encapsulated TLC's tracers have been applied to measure both temperature and velocity flow fields. We found that light scattered by the capsule shell inevitably diminishes saturation of the observed colours. Because in a slow motion the stress effects are negligible we prefer application of fine dispersed raw material in flow measurements.

\subsection{Surface Temperature Measurements}

Before the execution of a thermal or flow visualisation experiment, we should recognise the characteristics of the overall combination of the TLC, the light source, the optical and camera system, and make a rational plan for the total measurement system. The relationship between the temperature of the crystal and the measured Hue of the reflected light defines the calibration curve for the liquid crystal. The result is a curve relating the Hue of the reflected light to the surface temperature. A known 
temperature distribution exists on a "calibration plate" (brass plate) to which is attached the liquid crystal layer. In order to maintain a linear temperature distribution with desired temperature gradients, one end of a brass plate was cooled by stabilised water and the other end controlled electrically to give a constant temperature $[3,12,13]$. The brass plate with the liquid crystal layer is calibrated in place in the wind tunnel with the same lighting level and viewing angle used during the data acquisition phase of the experiment. The distribution of the colour component pattern on the liquid crystal layer was measured by RGB colour camera and a series of images at different temperatures defines the calibration.

A representative calibration curves including Hue and temperature distribution along the plate are shown in Figures 5 and 6. The analysis of calibration curves showed that halogen lamps are more suitable for LTC measurements [14]. In this particular experiment uncertainty was estimated as about $\pm 0.05^{\circ} \mathrm{C}$ by considering only the section of the surface used in the experiment, span-wise non-uniformities in Hue value are minimized.

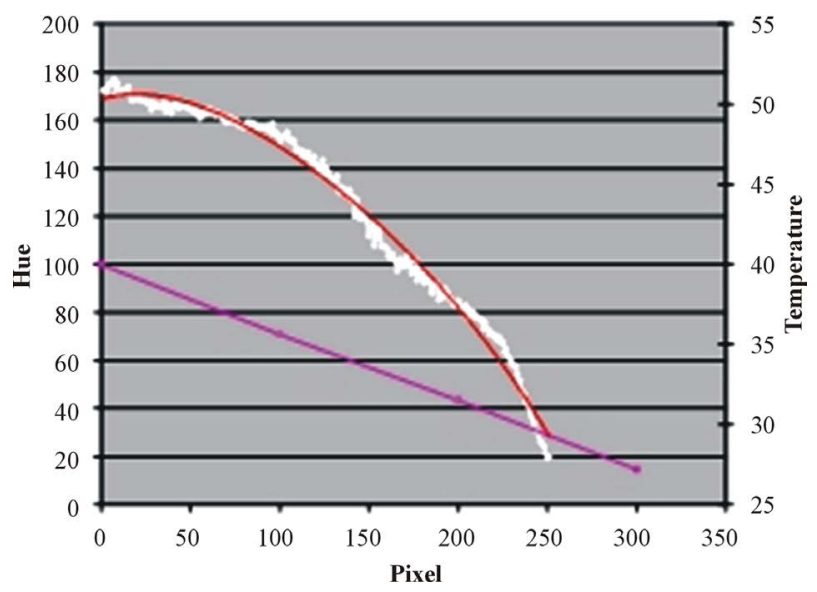

Figure 5. Representative calibration curve-Hue and temperature distribution along the test plate-halogen lamp.

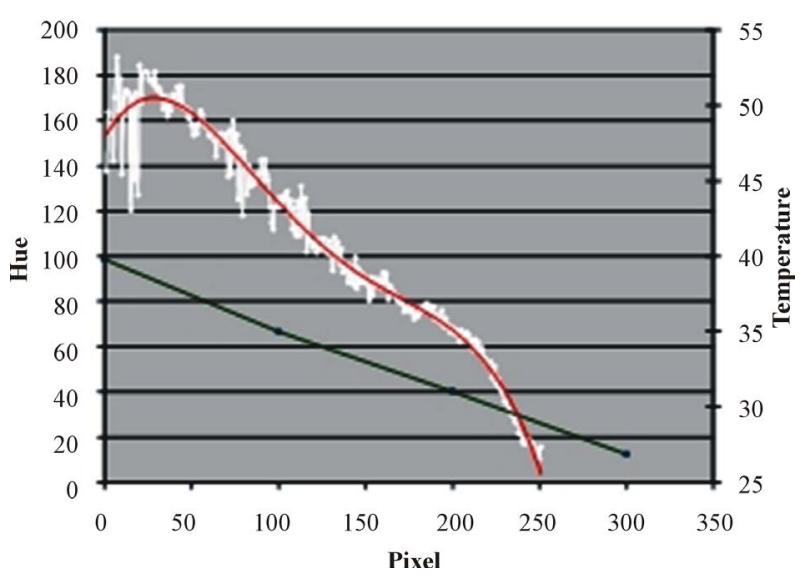

Figure 6. Representative calibration curve-Hue and temperature distribution along the test plate-tungsten lamp.
For the surface temperature measurement HSI colour image processing is utilised. These include image transformations, enhancement, analysis, compression, transformation and restorations.

Two main methods of surface temperature measurement are performed involving steady state and transient techniques. A brief history of these is given by Baughn $e t$ al. [15]. Recent reviews of heat transfer measurements have also been produced by Akino [12], Moffat [16,17], Jones et al. [18], Ashforth-Frost et al. [19] and Stasiek [13].

1) Steady State Analyses-Constant Flux Method

The steady state techniques employ a heated model and the TLC is used to monitor the surface temperature. Usually a surface electric heater is employed such that the local flux, $q$ is known and this, together with the local surface temperature, $T_{w}$, (found from the TLC), gives the local heat transfer coefficient, $h$,

and

$$
\begin{gathered}
q=I^{2} r \\
h=q /\left(T_{a}-T_{w}\right)
\end{gathered}
$$

where $T_{a}$ is a convenient driving gas temperature, $I$ is the current and $r$ is the electrical resistance per square of the heater.

2) Steady State Analyses-Uniform Temperature Method

The TLC-coated test specimen forms one side of a constant temperature water bath and is exposed to a cool/ hot air flow. The resulting thermograph is recorded on film or video and further measurement positions are obtained by adjusting the water bath temperature. This method is more time consuming due to the large volume of water that needs to be heated. In this case, the heat transfer coefficient is determined by equating convection to the conduction at the surface,

$$
h=\left[k\left(T_{w}-T_{b}\right)\right] /\left[x\left(T_{a}-T_{w}\right)\right]
$$

where, $T_{b}$ is a water-side temperature of the wall, $x$ the wall thickness and $k$ the thermal conductivity.

3) Transient Method

This technique requires measurement of the elapsed time to increase the surface temperature of the TLCcoated test specimen from a known initial temperature predetermined value. The rate of heating is recorded by monitoring the colour change patterns of the TLC with respect to time. If the specimen is made from a material of low thermal diffusivity and chosen to be sufficiently thick, then the heat transfer process can be considered to be one-dimensional (1-D) in a semi-infinite block. Numerical and analytical techniques can be used to solve the 1-D transient conduction equation. The relationship between wall surface temperature, $T$, and heat transfer coefficient, $h$, for the semi-infinite case is, 


$$
\left(T-T_{i}\right) /\left(T_{a}-T_{i}\right)=1-\exp \left(\beta^{2}\right) \operatorname{erfc}(\beta)
$$

and

$$
\beta=h(t / \rho c k)^{0.5}
$$

where, $\rho, c$ and $k$ are the model density, specific heat and thermal conductivity. $T_{i}$ and $T_{a}$ are the initial wall and gas temperatures and $t$ is time from initiation of the flow, Baughn et al. [15] and Jones et al. [18]. More recently Leiner et al. [20] developed a new formula for evaluation of heat transfer coefficient $h$ in the following form:

$$
h=-[\delta \rho c / t] \ln \left[\left(T_{a}-T\right) /\left(T_{a}-T_{i}\right)\right]
$$

where, $\delta$ is a plate wall thickness and the transient local surface temperature $T$ is detected after a time interval $t$.

\subsection{Full Field Temperature Measurements}

The temperature visualization is based on the property of some cholesteric and chiralnematic liquid crystal materials to refract light of selected wavelength as a function of the temperature and viewing angle. The response time of TLCs equals about $3 \mathrm{~ms}$. It is short enough for typical thermal problems in fluids. In the experimental realization the investigated flow has to be illuminated by a light sheet. The arrangement is similar to that used for classical PIV experiments, however white light is necessary to obtain selected colour refraction from the TLC particles. The colour of light refracted by TLCs depends not only on temperature but also on the observation angle. Therefore, it is important that the investigated flow is illuminated by a well-defined light plane and observed by a camera from a fixed direction. Density of the TLC material is very close to that of water and in most cases the TLC tracers can be treated as neutrally buoyant. Figure 7 demonstrates the application of un-encapsulated TLC tracers for visualization of natural convection in a differentially heated cube-shaped cavity. The hue (chromaticity) represents the dominant wavelength of the colour, i.e. the value that depends directly on the TLC's temperature. The temperature is determined by relating the hue to a temperature calibration function. The 8-bit representation of the hue value assures resolution better than $1 \%$.

However, the colour-temperature relationship is strongly non-linear (comp. Figure 8). Hence, the accuracy of the measured temperature depends on the colour (hue) value, and varies from $3 \%$ to $10 \%$ of the full colour
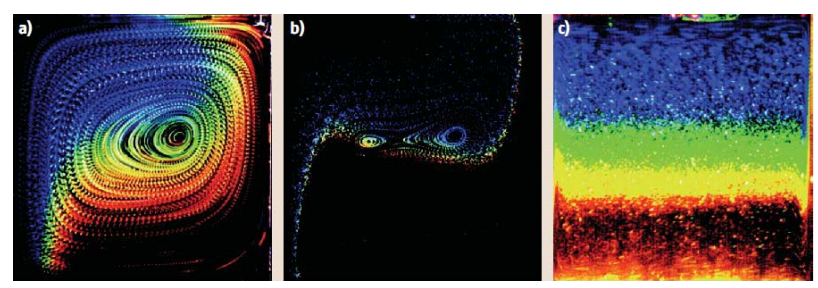

Figure 7. Natural convection in a differentially heated box.

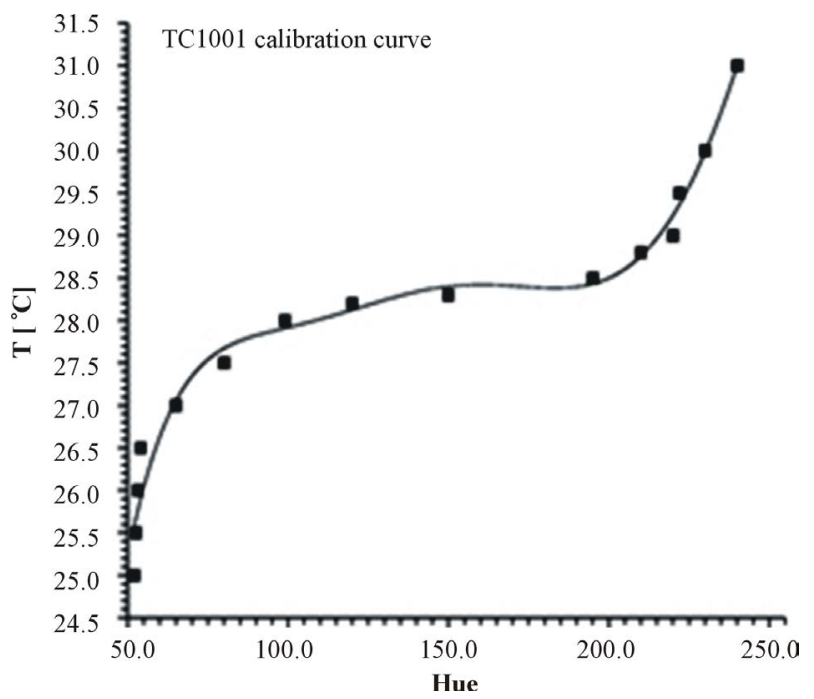

Figure 8. Temperature vs. hue for TLCs sample-natural convection of water. Calibration curve obtained by $6^{\text {th }}$ order polynomial fitted to the experimental points.

play range. For the liquid crystals typically used it results in an absolute accuracy of $0.15^{\circ} \mathrm{C}$ for lower temperatures (red-green colour range) and $0.5^{\circ} \mathrm{C}$ for higher temperatures (blue colour range). The most sensitive region is the colour transition from red to green and takes place for a temperature variation less than one Celsius degree. The 2-D velocity vector distribution has been measured by digital particle image velocimetry. By this method, the motion of the scattering particles, observed in the plane of the illuminating light sheet, is analyzed. For this purpose, the colour images of the TLC tracers are transformed to black \& white intensity images. To improve contrast and the visibility of particles a special filtering technique is applied. It allows obtaining bright images of the tracers, well suited for PIV. The cross-correlation analysis performed between different images of the sequence (time interval between pairs changes) allows us to preserve similar accuracy for both the low and high velocity flow regions. For a typical displacement vector of 10 pixels the relative accuracy of the velocity measurement (for single point) is better than $6 \%$.

The typical experimental setup used for the flow measurements $[9,10]$ consists of a convection box, a halogen tube lamp, the 3-chip CCD colour camera and the 32-bit frame grabber. The flow field is illuminated with a $2 \mathrm{~mm}$ thin sheet of white light from a specially constructed halogen lamp or xenon-flash tube and observed in the perpendicular direction. The 24-bit colour images, typically of $768 \times 564$ pixels, have been acquired with a personal computer. Using a PCI based colour frame grabber (AMSTD ITI) and a 128 MB Pentium computer, our setup permits us to gain in real time over 50 RGB images, before they have to be saved on the computer magnetic disk. 
Three different flow regimes are visualized using unencapsulated liquid-crystal tracers: Figure 7(a) Low Rayleigh number (104): temperature difference between walls $4^{\circ} \mathrm{C}$, TLC material TM107, box size $38 \mathrm{~mm}$; Figure 7 (b) Intermediate Rayleigh number $(8 \times 104)$ : temperature difference $16^{\circ} \mathrm{C}$, TLC material TM445, box size 38 mm; Figure 7(c) High Rayleigh number $(1.3 \times$ 108): temperature difference $34^{\circ} \mathrm{C}$, TLC material TM317, box size $80 \mathrm{~mm}$. Images (a) and (b) were obtained from color slides exposed 20 times every 15 s; image (c) was created from 20 digital images taken every $200 \mathrm{~ms}$ and added in a computer memory [21].

\section{Application Experiments}

In order to demonstrate the feasibility of TLC techniques in practical heat transfer contexts the authors have performed several experiments.

\subsection{Surface Measurements-Selected Results}

The experimental study was carried out using an open low-speed wind tunnel consisting of entrance section with fan and heaters, large settling chambers with diffusing screen and honeycomb, and then mapping and working sections. Air is drawn through the tunnel using a fan able to give Reynolds numbers of between 500 and 50,000 in the mapping and working sections. The working air temperature in the rig ranges between $25^{\circ} \mathrm{C}$ to $65^{\circ} \mathrm{C}$ produced by the heater positioned just downstream of the inlet. The major construction material of the wind tunnel is perspex. Local and mean velocity are measured using conventional Pitot tubes and DISA hot-wire velocity probe. The alternative effects of constant wall temperature and constant heat flux boundary conditions are obtained using a water bath, while the temperature is controlled with a thermostat capable of establishing and maintaining temperature to within $\pm 0.01^{\circ} \mathrm{C}$ accuracy. Photographs are taken using a RGB video-camera and true-colour image processing system. The heat transfer coefficient is a defined quantity, calculated from the surface heat flux and the difference between the surface temperature and some agreed reference temperature. This is usually the far field temperature, the mixed mean temperature or the adiabatic surface temperature. Liquid crystals can be used to determine the distribution of the surface temperature, and if the surface heat flux can be found, this allows evaluation of the heat transfer coefficient or the Nusselt number. The alternative effects of constant wall temperature and constant heat flux boundary conditions are obtained using a water bath. Photographs are taken using a RGB video-camera and a truecolour image processing technique. Usually several isotherms (each corresponding to a different heat flux) are taken by RGB video-camera to record the local Nusselt contours under an oblique Reynolds number. The locations of each isotherm and colour (adjusted to each Nusselt number) are digitised following a projection of the false colour image on a digitising image respectively (this particular method can be called "Image Combination Technique” ICT). Figure 9 shows photographs of the colour distribution of the liquid crystal layer around a square section column, image of the computer display after segmentation processing (Hue: 45 - 55) and false colour image processing (ICT) respectively on bottom.

Also Figure 10 shows a false colour image of local Nusselt number contours over a central diamond of the corrugated-undulated heat exchanger surfaces. The cross corrugated and corrugated-undulated surfaces are frequently employed to increase heat transfer coefficient for high heat flux applications.

Improvements in their flow and thermal characteristics do not require any demonstrations and would substantially reduce fuel and production costs. The measuring technique comprising the use of LC flexible sheets and true-colour processing may also be used for a great variety of applications and should be of considerable use in improving the design of all types of compact heat exchanger. Experimental procedures cover full-field flow patterns in classic heat exchanger elements (flat plate

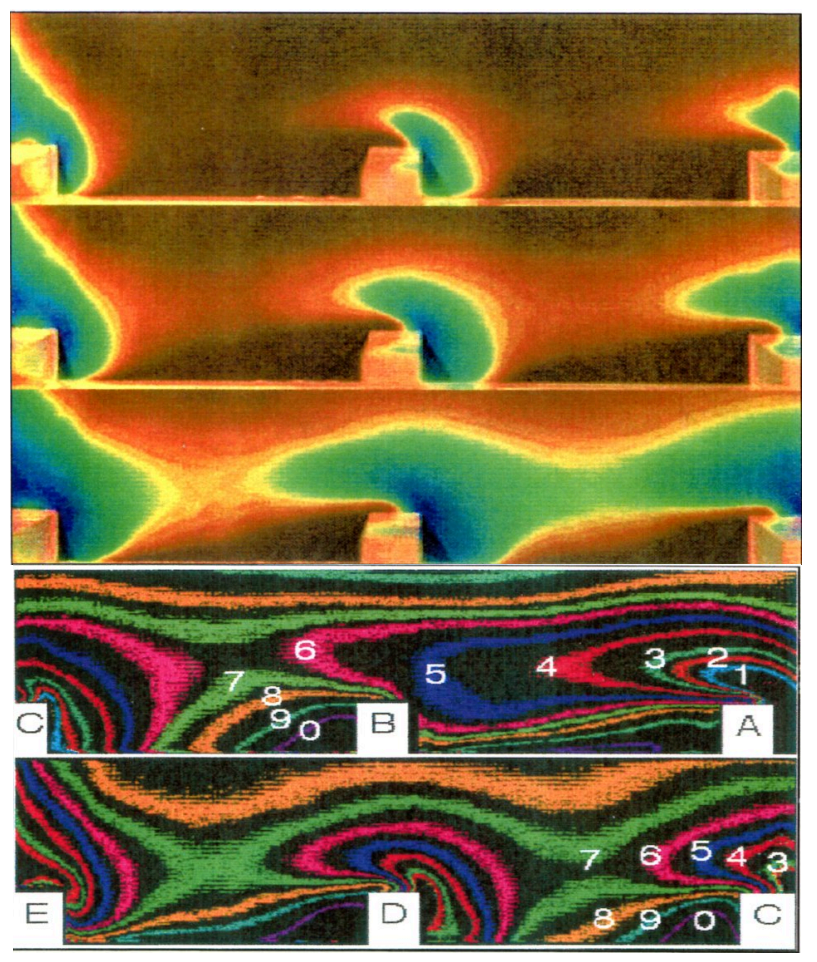

Figure 9. True-colour images from liquid crystal thermography for an endwall surface with in-line square ribs (up); Pattern of 10 Nusselt numbers $\mathrm{Nu}$ reconstructed by false colour images of the heat transfer surface for $\operatorname{Re}=20,000(0$ : $\mathrm{Nu}=79 ; 9$ : 99; 8: 113; 7: 123; 6: 136; 5: 147; 4: 160; 3: 175; 2: 185; 1: 209) (bottom). 
with fine-tubes in-line, staggered and with vortex generators) describing local heat transfer coefficient and Nusselt number on the surfaces. An example of such results is presented in Figure 11. Impinging jets associated with water jet, air-water spray and air jet are widely used to provide high local heat and/or mass transfer in various applications including metal rolling processes, glass manufacturing, paper drying or gas turbine cooling.

They offer the potential of high heat transfer rate and relative ease of controlling specific areas to be cooled (heated, dried, or moistened). In order to study the influence of thermo-mechanical properties and geometry of an oblique impinging jet on heating or cooling practices on/from plate, a good understanding of the heat and mass transfer aspects of the process is essential (Figures 12 and 13).

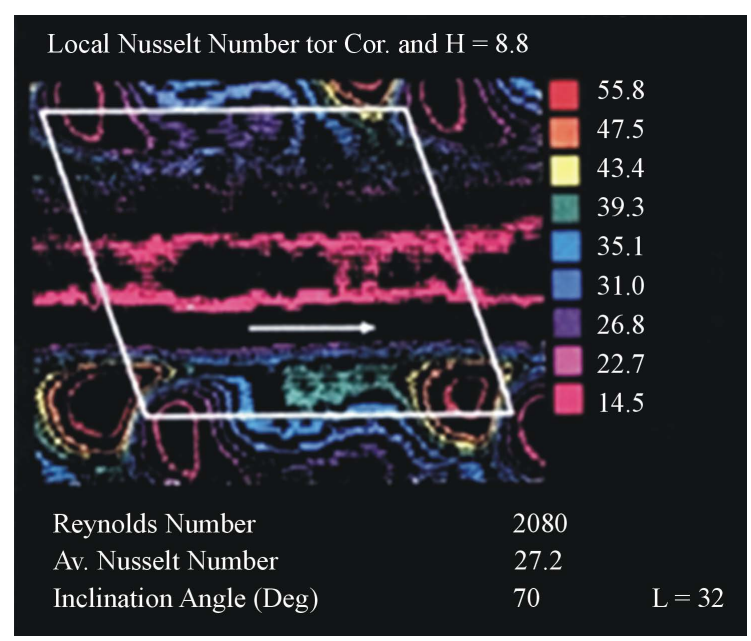

Figure 10. False colour image of local Nusselt number contours over a central diamond of the undulated plate of corrugated-undulated geometry $\left(\phi=70^{\circ}, \mathrm{Re}=2080, \mathrm{H}=8.8\right.$ $\mathrm{mm}, \mathrm{L}=32 \mathrm{~mm})$.

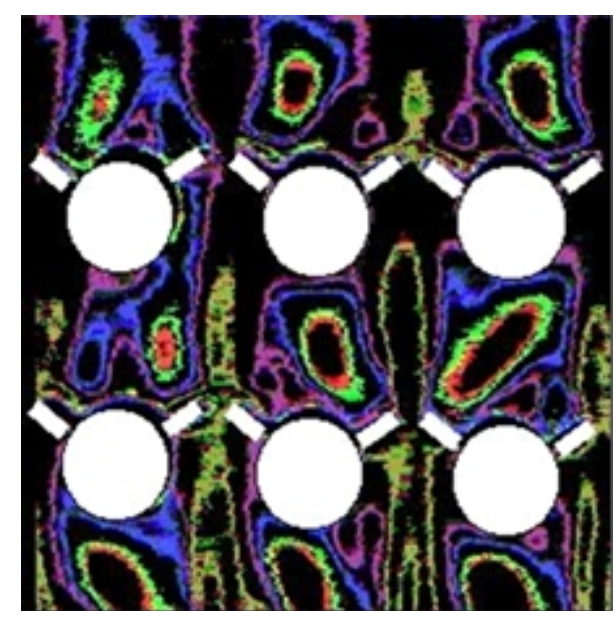

Figure 11. Reynolds number-1690; Average Nusselt number-14.5; Local Nusselt number: red-6.80, green-7.82, blue-11.98, violet-14.60, khaki-22.15.

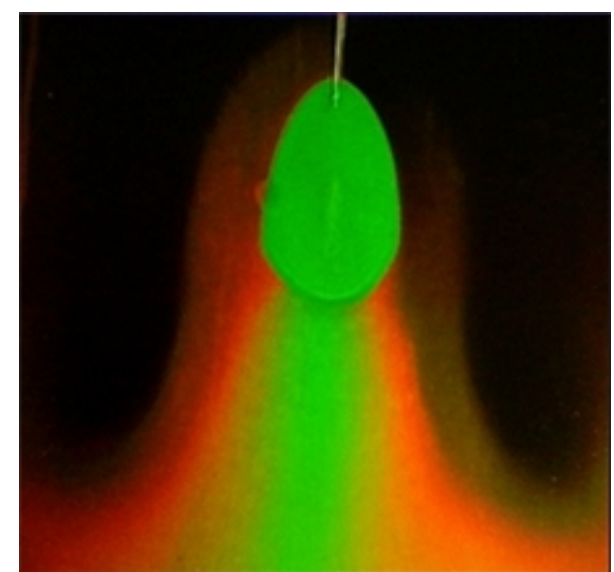

Figure 12. True-colour image of water impinging jet on a liquid crystal coated surface.

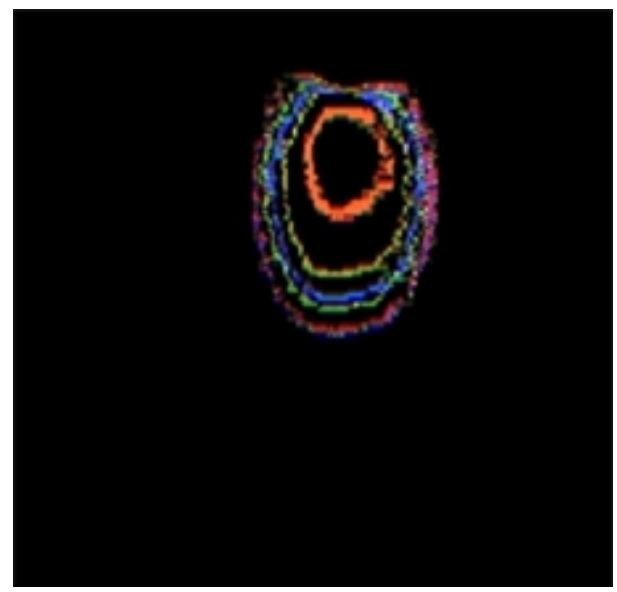

Figure 13. False colour image of local Nusselt number contours. Local Nusselt number: red-39.2, khaki-14.9, blue -11 , green-9.1, violet $-5.98, \mathrm{Nu}_{\mathrm{avr}}-15.03$.

\subsection{Natural Convection in a Closed Cavity-Selected Results}

Vertical temperature gradients are mainly responsible for the atmospheric or oceanographic fluid motion. Development of the nocturnal stable stratification over flat areas, inversion of temperature gradient and break-up of the convective boundary layer developed at the ground levels can be simulated using small-scale laboratory experiments.

One of the typical features characterizing instabilities generated by vertical temperature gradient are plumes or ejections appearing, when the thermal boundary layer breaks up. Figure 14 shows temperature and velocity visualization in glycerol-filled cavity under free convection.

Figure 15 presents visualization of natural convection in water close to the freezing point, using a hot (left) wall at a temperature of $10^{\circ} \mathrm{C}$ and a right wall at a temperature of $0^{\circ} \mathrm{C}$ for (a) and (b), $-10^{\circ} \mathrm{C}$ for (c). Three types of liq- 


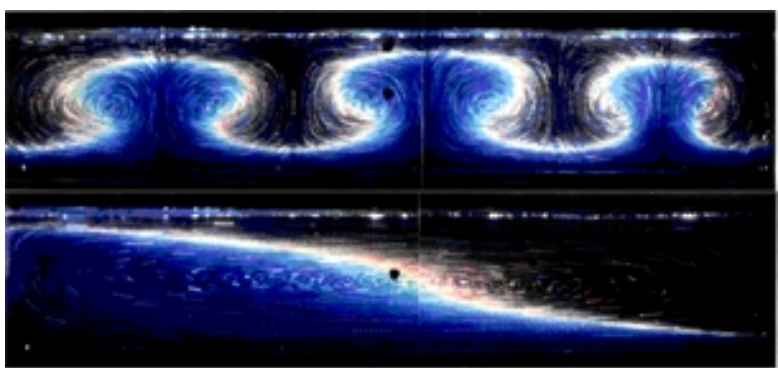

Figure 14. Temperature and velocity visualization in glycerol-filled cavity under free convection using chiralnematic liquid crystal $\left(\mathrm{Ra}=1.2 \times 10^{4}, \mathrm{Pr}=12 \times 10^{3}\right)$, horizontal position (up) and vertical position (bottom).
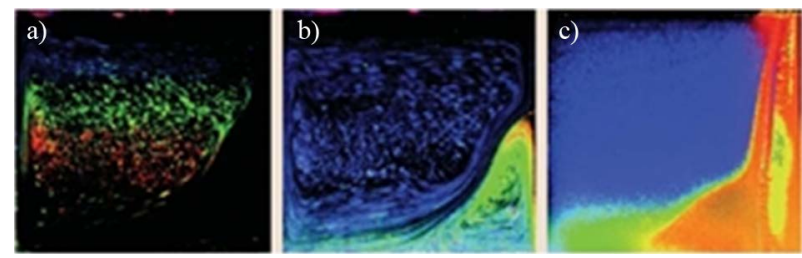

Figure 15. Visualization of natural convection in water close to the freezing point, using a hot (left) wall at a temperature of $10^{\circ} \mathrm{C}$ and a right wall at a temperature of $0^{\circ} \mathrm{C}$ for (a) and (b), $-10^{\circ} \mathrm{C}$ for (c).

uid-crystal tracers were used to indicate the temperature variation: 1) encapsulated TLCs BM100/R6C12W showing details of flow and temperature for normal circulation (on the left); sum of 10 images taken every $1 \mathrm{~s}$; 2) TLC mixture (TM445 and TM912) active at lower temperatures, visualizing the complex structure of abnormal circulation; sum of 20 images; 3) TLC mixture with a composition matched to the freezing point [21].

The formation of ice has been studied by decreasing the lid temperature to $-10^{\circ} \mathrm{C}$. A complicated flow pattern, is established, which becomes visible also in the structure of the ice surface. It was found that the creation of the ice layer at the lid has a stabilizing effect on the flow. Figure 16 shows the temperature and velocity field evaluated at the time step $3600 \mathrm{~s}$ for this case. It appears that even this quasi-steady result is only qualitatively well described by the numerical model. One of the possible reasons is strong sensitivity of this configuration to imposed thermal boundary conditions at the sidewalls [21].

\subsection{Medical Applications}

Liquid crystals in the mode of thermographic examination, can be also used in the case of diagnostics and monitoring of different diseases e.g.: diagnosis of cancers, tumours and inflammatory states (liver, lung, etc.), evolution of skin tests (allergy, tuberculin), traumatology and forensic medicine.

Those methods are especially useful for children because they are non-invasive, fast, reliable and visually attractive. Because the temperature of human skin de-

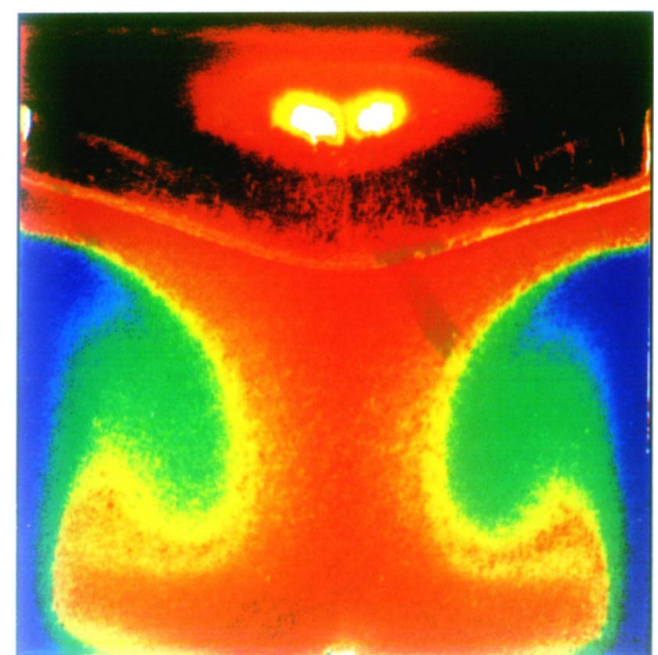

(a)

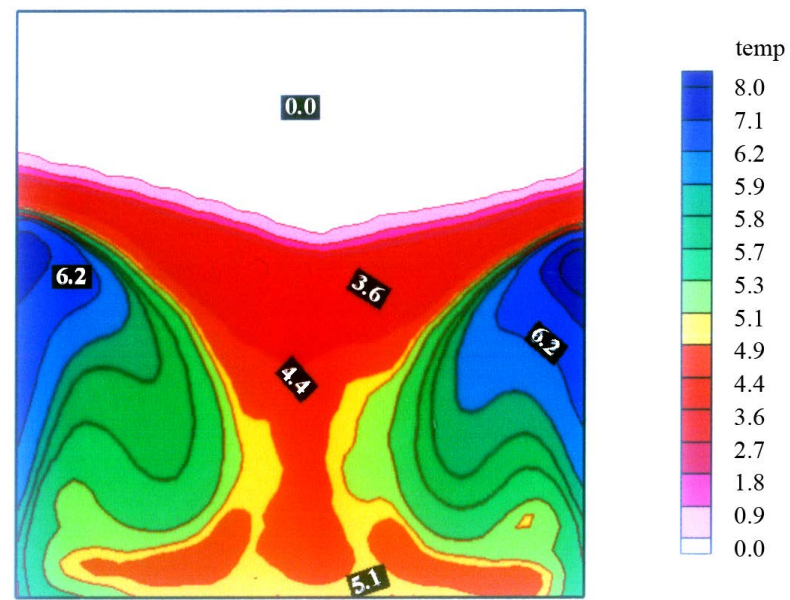

(b)

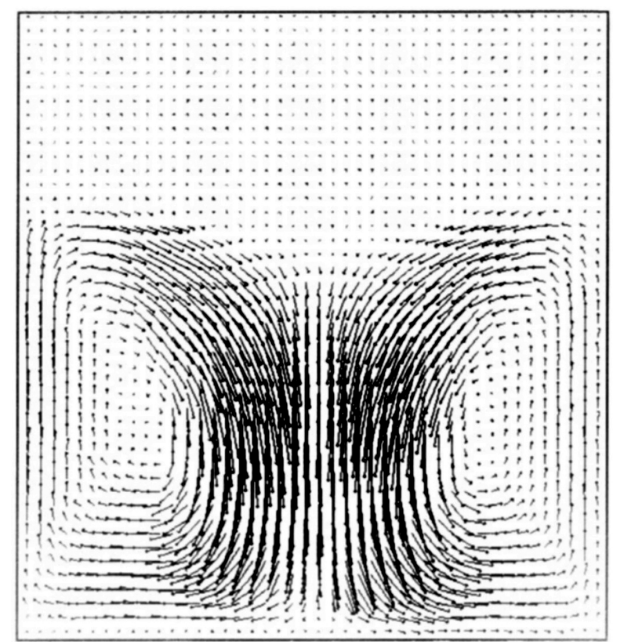

(c)

Figure 16. Freezing of water under a cold surface in a lid cooled cavity. Recorded image of TLC tracers (a), evaluated temperature (b) and velocity (c) fields. Time step-3600 s after cooling starts; Isothermal lid temperature $T_{c}=-10^{\circ} \mathrm{C}$, external temperature $\mathrm{T}=20^{\circ} \mathrm{C}$. 
pends upon the metabolic level of neighbouring tissues, heat transfer can occur via muscular and adipose tissues, blood circulation, muscular tensions, body position or effect of drugs. This kind of medical diagnosis should be confirmed by other independent methods in the case of diseases suspicion.

Requirements for thermographic examination are wellknown [22-24]. The minimum area of a testing room has to be 9 square metres. All devices emitting heat should be removed, if possible. Air movements have to be minimised. The preferred temperature of the testing room should be $25^{\circ} \mathrm{C}$ to $27^{\circ} \mathrm{C}$, while humidity should be $50 \%$ to $70 \%$. Both these parameters should be carefully controlled during measurement. The effect of sunlight should be minimised. Light sources have to secure scattered light of low intensity to avoid light reflections. Patients should abstain from physical activity during 20 minutes before measurement. The skin area studied should be exposed for at least 15 minutes before a test to ensure thermal equilibrium with the environment.

The patient's position should be similar to their physiological (normal) one to avoid temperature changes due to enforced changes of blood circulation. Body areas studied should not contact other body areas. Medicines, drugs (alcohol, nicotine and so on) and treatments affecting blood circulation should not be taken, if possible, for 24 hours before measurement. Also patients should not have cold and hot drinks and not eat large meals before measurement. Cosmetics can also affect thermal properties of skin. All subsequent measurements have to be done at the same hour of the day.

The thermological report should contain all conditions of measurement, including the states of the patient and environment. The patient should be allowed to see the thermographic images because their colours have a positive psychological effect especially in the case of children. Various cases have been studied and typical results are shown in Figures 17-19 [24].

\subsection{Augmented Heat Transfer-Recent Developments at GUT}

Wind tunnel with working section featuring transverse and inclined vortex generators has been used to study the air flow. In the accomplished experiments, the Reynolds number was varied from 9000 to 35,500 . The aspect ratio of the rectangular channel was equal to 6.37 and pitchto-height-ratio of the ribs was equal to 11 . Both, steadystate and transient liquid crystal techniques, were employed in the research to determine the distribution of surface temperature and subsequent evaluation of heat transfer coefficient.

Air is drawn through the tunnel using a fan able to attain the Reynolds numbers in the range from 500 to 40,000 . The working air temperature in the rig ranges

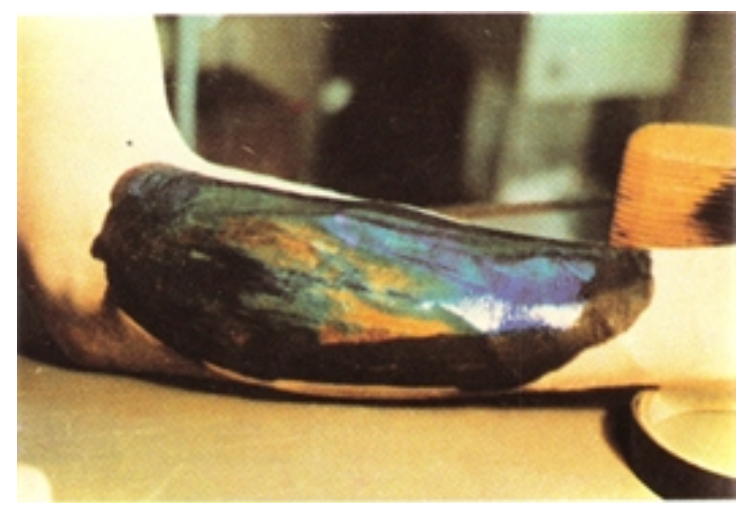

Figure 17. Thermogram of forearm.

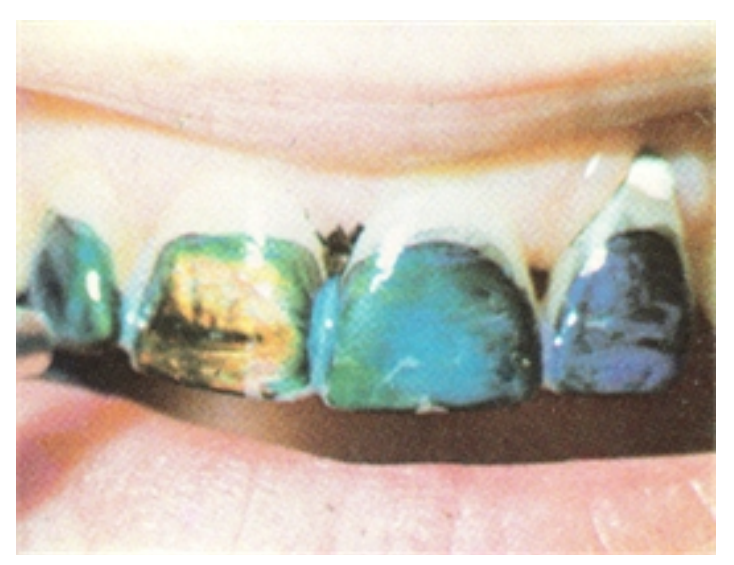

Figure 18. Thermogram of teeth.

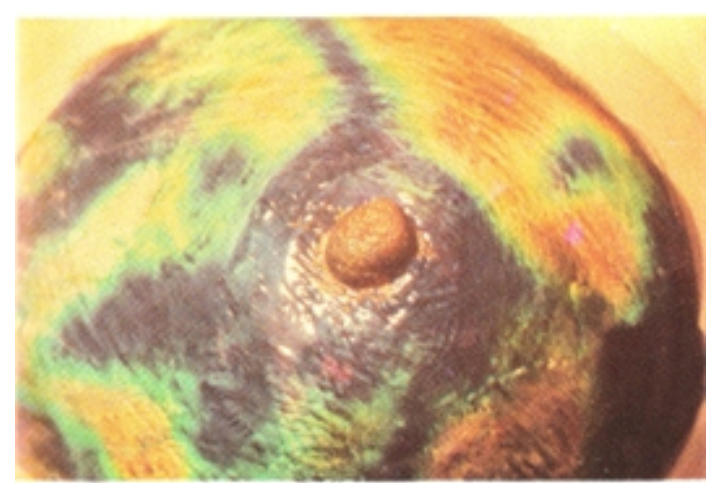

Figure 19. Thermogram of breast cancer (dark region).

from $15^{\circ} \mathrm{C}$ to $65^{\circ} \mathrm{C}$ produced by the heater or cooler positioned just downstream of the inlet. The major construction material of the wind tunnel is perspex. Local and mean velocity are measured using conventional Pitot tubes and DISA hot-wire velocity probe.

The alternative effects of constant wall temperature and constant heat flux boundary conditions are obtained using plate electric heater [25]. Photographs are taken using RGB video-camera and a true-color image processing technique. The liquid crystals used here, manufactured in sheet form by Merck Ltd. [26], had an event 
temperature range from $30.0^{\circ} \mathrm{C}$ to $35.0^{\circ} \mathrm{C}$. In this particular experiment uncertainty for temperature measurement was estimated at about $\pm 0.05^{\circ} \mathrm{C}$ by considering only the section of the surface used in the experiment, span-wise non-uniformities in Hue value are minimized.

In many cases, as mentioned above, remarkable enhancement of local and spatially averaged surface heat transfer rates are possible with rib turbulators, in spite of the lower local Nusselt number at certain locations along the ribbed surfaces. Prior to ribbed turbulator test section is a $255 \times 40 \mathrm{~mm}$ inlet duct that is $550 \mathrm{~mm}$ in length. This is equivalent to 7.96 hydraulic diameter (where the hydraulic diameter is $69.11 \mathrm{~mm}$ ). The test surface that is analyzed contains a collection of rib turbulators that are perpendicular and angled with respect to the flow stream (Figure 20). To determine the surface heat flux (used to calculate heat transfer coefficients and Nusselt numbers), the convective power levels provided by the thermofoil heaters are divided by flat test surface area.

Spatially resolved temperature distributions along the bottom rib turbulator test surface are determined using liquid crystals thermography (LCT) and true-color image processing system commercially available from Data Translation Inc. [1].

The heat transfer coefficient is one of the most relevant parameters in the heat exchangers. This section presents measurement results for this parameter.

In the discussion that follows, the Nusselt number

$$
\mathrm{Nu}=h \cdot D_{h} / k
$$

where $h$ is the heat transfer coefficient, $D_{h}$ is the channel hydraulic diameter and $k$ is the molecular thermal conductivity of air. The heat transfer coefficient is then based on the flat projected area and is determined using

$$
h=q_{n} /\left(T_{w}-T_{\alpha}\right)
$$

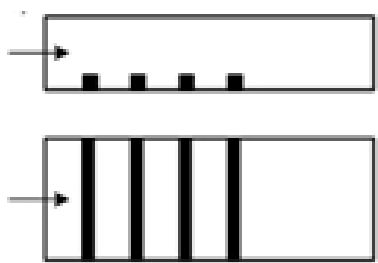

(a)

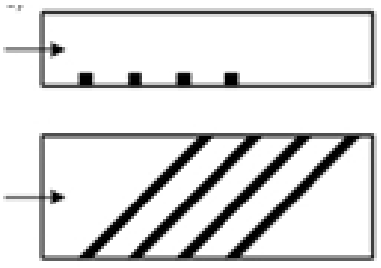

(c)
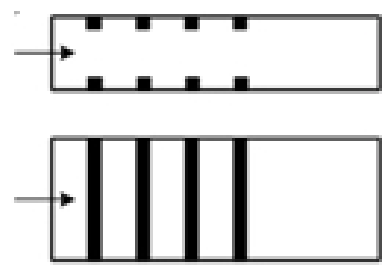

(b)

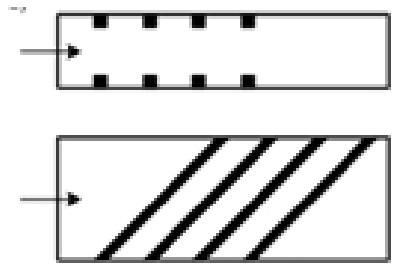

(d)
Figure 20. Four types of transverse vortex generators (cross-corrugated and rectangular ribs). where $q_{n}$ is the local (net) surface heat flux, local surface temperature $T_{w}$ (found from LCT) and $T_{\alpha}$ is the timeaveraged, local mixed mean air temperature.

The surface Nusselt number distribution along the rib turbulator test surface for four Reynolds number are presented in Figures 21 and 22 for two ribs geometries (a) and (b). Geometry (b) gave the best overall heat transfer enhancement. More results can be found in [27]. Transverse vortex generators enhance heat transfer by several hundred percent but only for certain Reynolds numbers. Comparison between the maps of velocity vectors and local Nusselt numbers contours reveals that high velocity do not enhance heat transfer in some cases, probably the moving fluid (with high velocity) does not penetrates into the boundary layer and flows above the ribs. Opti-

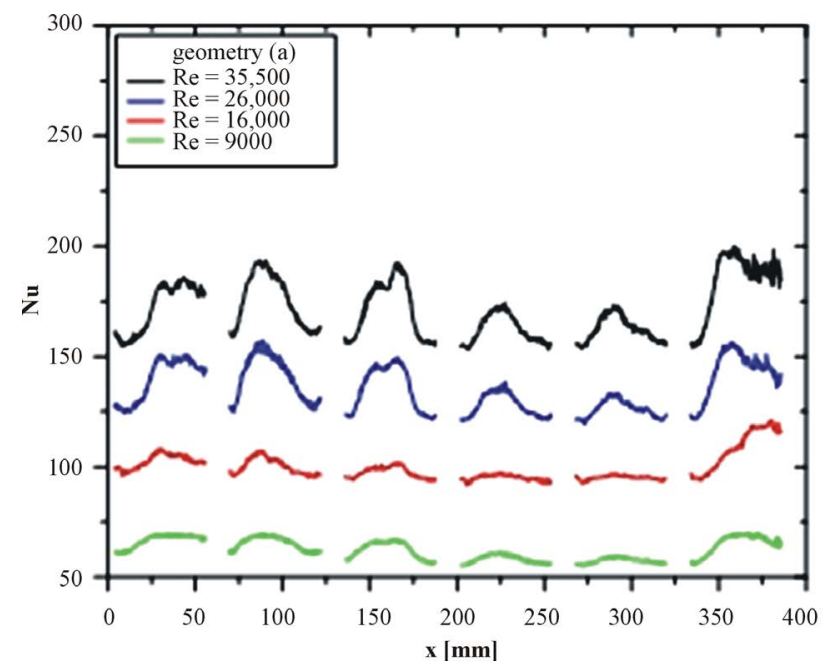

Figure 21. Nusselt number distribution in consecutive test sections for different Reynolds numbers $(\operatorname{Re}=9000,16,000$, 26,000 and 35,500 ) taken by steady-state method measurements for geometry (a).

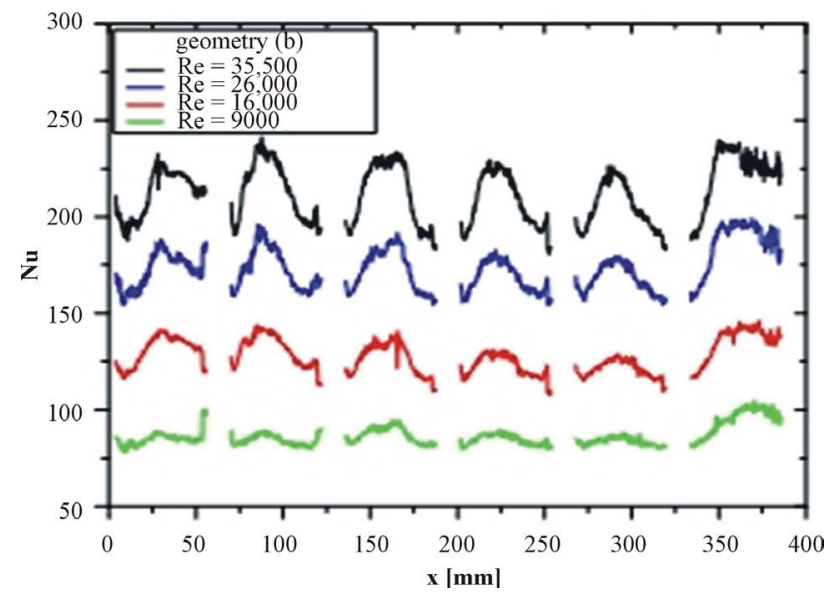

Figure 22. Nusselt number distribution in consecutive test sections for different Reynolds numbers $(\operatorname{Re}=9000,16,000$, 26,000 and 35,500 ) taken by steady-state method measurements for geometry (b). 
mum flow condition should be established for high efficient enhancement of heat transfer augmentation in particular environment e.g. flow and heat exchanger configuration.

Figures 23 and 24 show results of transient method experiments for geometry (a) and geometry (b). The local heat transfer coefficient and then local Nusselt number was determined from Leiner et al. formula-Equation (10).

Table 1 presents average Nusselt number distribution as well as average heat transfer coefficient taken between $3^{\text {rd }}$ and $4^{\text {th }}$ rib for each geometry for both steady-state and transient methods. For the purpose of comparison, calculations for the baseline condition of a smooth channel (without ribs) with asymmetric heating were performed. The local Nusselt number was normalized by the Nusselt number for fully developed turbulent flow in smooth circular tubes given by the well known Dittus-Boelter correlation (Equation (13)).

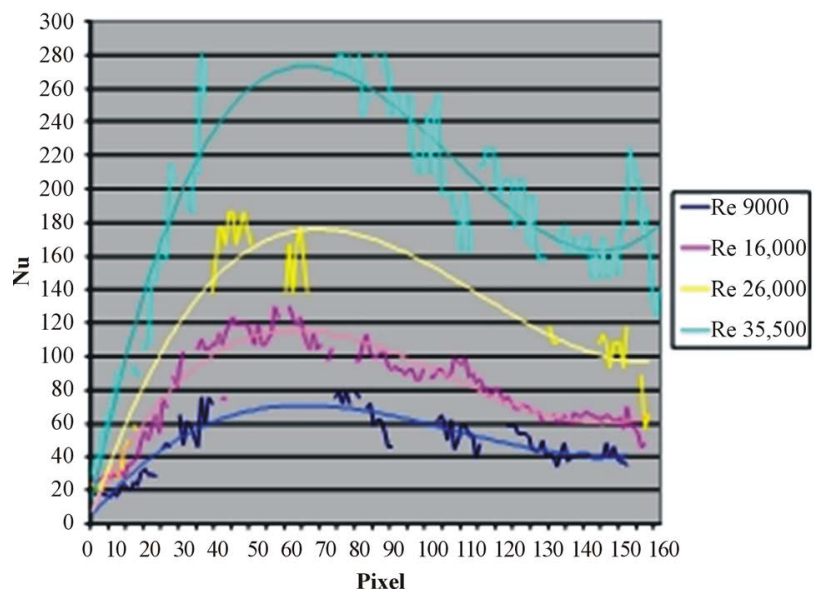

Figure 23. Nusselt number distribution in consecutive test sections for different Reynolds numbers $(\operatorname{Re}=9000,16,000$, 26,000 and 35,500 ) taken by transient method measurements for geometry (a).

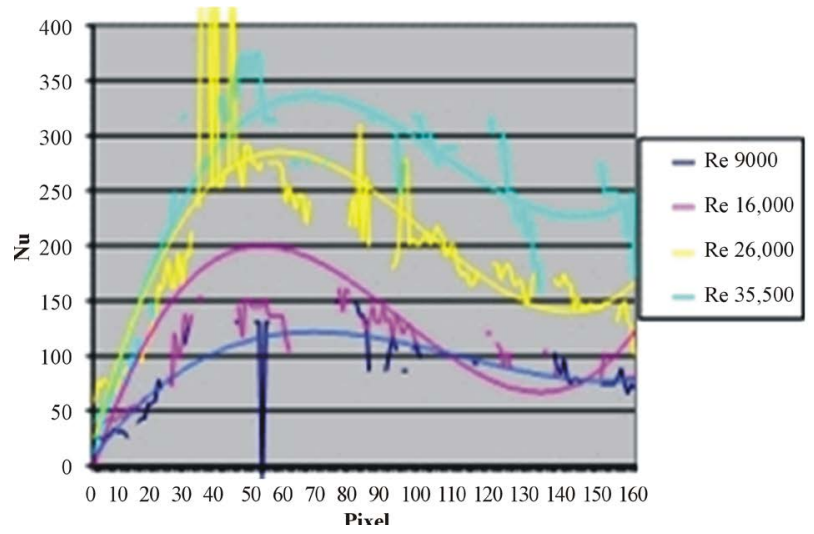

Figure 24. Nusselt number distribution in consecutive test sections for different Reynolds numbers taken by transient method measurements $(\operatorname{Re}=9000,16,000,26,000$ and 35,500) for geometry (b).
Table 1. Average Nusselt number and heat transfer coefficient distribution for different ribs geometry.

\begin{tabular}{|c|c|c|c|c|}
\hline Geometry & Re & $\mathrm{Nu}_{\text {AV_STEADY-STATE }}$ & $\mathrm{Nu}_{\mathrm{AV} \_ \text {TRANSIENT }}$ & $\mathrm{Nu}_{0}$ \\
\hline \multirow{4}{*}{ (a) } & 9000 & 62.34 & 47.33 & 29 \\
\hline & 16,000 & 97.51 & 82.01 & 46 \\
\hline & 26,000 & 135.55 & 111.30 & 68 \\
\hline & 35,500 & 171.68 & 188.06 & 87 \\
\hline \multirow{4}{*}{ (b) } & 9000 & 87.09 & 83.40 & 29 \\
\hline & 16,000 & 126.37 & 132.97 & 46 \\
\hline & 26,000 & 172.25 & 198.08 & 68 \\
\hline & 35,500 & 211.62 & 251.34 & 87 \\
\hline \multirow{4}{*}{ (c) } & 9000 & 60.88 & 72.94 & 29 \\
\hline & 16,000 & 86.14 & 106.59 & 46 \\
\hline & 26,000 & 121.74 & 157.68 & 68 \\
\hline & 35,500 & 156.67 & 198.23 & 87 \\
\hline \multirow{4}{*}{ (d) } & 9000 & 60.17 & 80.44 & 29 \\
\hline & 16,000 & 86.22 & 114.18 & 46 \\
\hline & 26,000 & 129.21 & 189.57 & 68 \\
\hline & 35,500 & 161.59 & 241.95 & 87 \\
\hline
\end{tabular}

$$
\mathrm{Nu}=0.023 \cdot \mathrm{Re}^{0.8} \cdot \operatorname{Pr}^{0.4}
$$

Nusselt number for smooth channel calculated according to Equation (13) for given Reynolds numbers e.g. 9000, $16,000,26,000$ and 35,500 results in the following data respectively: 29, 46, 68 and 87. Normalized Nusselt number, $\mathrm{Nu} / \mathrm{Nu}_{0}$, are presented in Figures 25 and 26 as functions of the Reynolds number for all five ribs configurations. The heat transfer augmentation is in the $1.79 \mathrm{e} 2.92$ range and decreases generally with $\mathrm{Re}$, as typically occurs in the ribroughened channels [28,29]. It is the evident that transverse vortex generators enhance heat transfer as much as a few times the base value but only for certain Reynolds numbers.

Results of experiments performed with transient method give similar results in terms of average Nusselt numbers and the best performing geometry is (b). However there are small differences regarding other geometries. The steady-state method is more reliable however it more expensive.

\section{Conclusion}

True-colour image processing of liquid crystal patterns allows new approaches to old problems and at the same time opens up new areas of research. Processed image data make available quantitative and full-field information about the distribution of temperature and heat transfer 


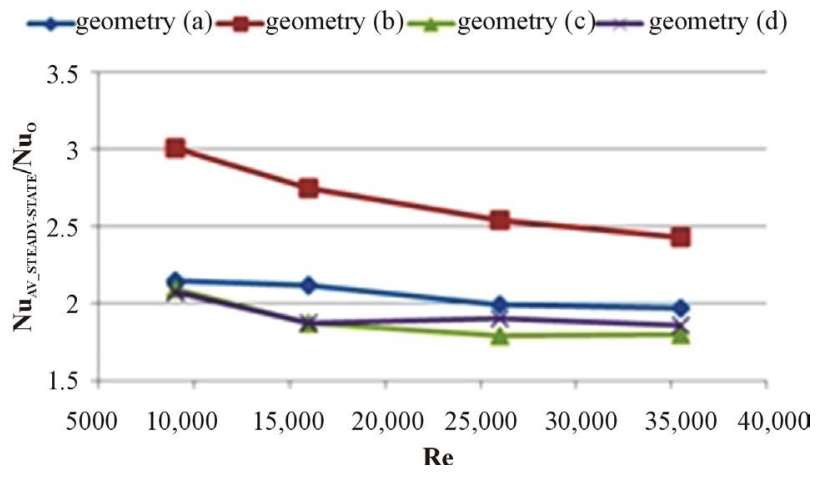

Figure 25. Normalized Nusselt number as a function of Reynolds number for different ribs geometry taken from steadystate method.

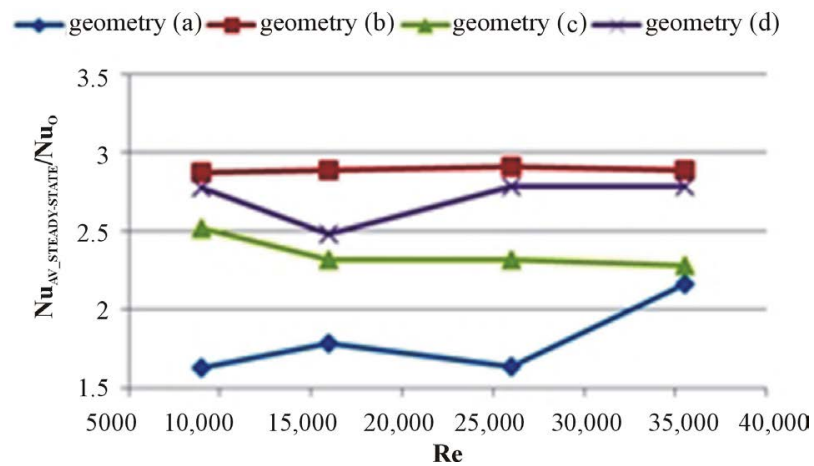

Figure 26. Normalized Nusselt number as a function of Reynolds number for different ribs geometry taken from transient method.

coefficient which will undoubtedly encourage the study of situations which have been, until now, too complex to consider. The measuring technique comprising the use of LC flexible sheets and true-colour processing may also be used for a great variety of applications and should be of considerable use in improving the design of all types of rotary and compact heat exchangers. Computer aided analysis of colour images of unsealed TLC-tracers is a useful non-invasive method of investigating three-dimensional flow and temperature fields and facilitates their easy comparison with numerical results.

\section{REFERENCES}

[1] DATA TRANSLATION Ltd., "Image Processing Handbook,” 1991.

[2] M. Travis, "True-Colour Image Processing on the Desktop,” Photonics Spectra, Laurin Publishing Co., Inc., Pittsfield.

[3] J. Stasiek and M. W. Collins, "The Use of Liquid Crystals and True-Colour Image Processing in Heat and Fluid Flow Experiments," Atlas of Visualization, Vol. 2, CRC Press, Inc., New York, 1996.

[4] J. Stasiek, G. Tanda and M. Ciofalo, "Heat Transfer Measurement in Heat Exchangers by Liquid Crystal Thermo- graphy," Euromech406 Colloquium, Warsaw, 6-8 May 1999, Book of Abstracts IPPT PAN.

[5] J. Stasiek, A. Stasiek, M. Jewartowski and M. W. Collins, "Liquid Crystal Thermography and True-Colour Digital Image Processing,” Optics \& Laser Technology, Vol. 38, No. 4, 2006, pp. 243-256.

http://dx.doi.org/10.1016/j.optlastec.2005.06.028

[6] M. Parsley, "The Use of Thermochrome Crystals in Heat Transfer and Fluid Flow Visualization Research," FLUCOME'88, Sheffield University, 5-9 September 1988, pp. 216-220.

[7] R. Reinitzer, "Beitrage zur Kenntniss des Cholestrins," Monatschefte für Chemie, Vol. 9, 1888, pp. 421-441.

[8] W. J. Hiller, St. Koch, T. A. Kowalewski and F. Stella, "Onset of Natural Convection in a Cube," International Journal of Heat and Mass Transfer, Vol. 36, No. 13, 1993, pp. 3251-3263. http://dx.doi.org/10.1016/0017-9310(93)90008-T

[9] J. A. Stasiek and T. A. Kowalewski, "Thermocromic Liquid Crystals Applied for Heat Transfer Research,” OptoElectronics Review, Vol. 10, No. 1, 2002, pp. 1-10.

[10] T. A. Kowalewski, A. Cybulski and M. Rebow, "Particle Image Velocimetry and Thermometry in Freezing Water," G. M. Carlomagno and I. Grant, Eds., 8th International Symposium on Flow Visualization, Sorrento, 1-4 September 1998, pp. 24.1-24.8.

[11] T. A. Kowalewski and M. Rebow, "Freezing of Water in the Differentially Heated Cubic Cavity,” International Journal of Computational Fluid Dynamics, Vol. 11, No. 3-4, 1999, pp. 193-210. http://dx.doi.org/10.1080/10618569908940874

[12] N. Akino, T. Kunugi, Y. Shiina, K. Ichimiya and A. Kurosawa, "Fundamental Study on Visualization of Temperature Fields Using Thermosensitive Liquid Crystals," In: R. Reznicek, Ed., Flow Visualization V, Hemisphere Publishing Corp., Washington DC, 1990, pp. 87-92.

[13] J. Stasiek, "Thermochromic Liquid Crystals and True Colour Image Processing in Heat Transfer and Fluid Flow Research,” Journal of Heat and Mass Transfer, Vol. 33, No. 1-2, 1997, pp. 27-29.

[14] A. Stasiek, "Heat Transfer Enhanced in a Rectangular Channel with Varying Number of Ribbed Walls," Ph.D. Thesis, Technical University of Koszalin, Koszalin, 2008.

[15] J. W. Baughn and X. Yan, "Liquid Crystal Methods in Experimental Heat Transfer," Proceedings of 32nd Heat Transfer and Fluid, Mechanics Institute Sacramento, California, 1991, pp. 15-40.

[16] R. J. Moffat, "Describing the Uncertainties in Experimental Results,” Experimental Thermal and Fluid Sciences, Vol. 1, No. 1, 1988, pp. 3-17. http://dx.doi.org/10.1016/0894-1777(88)90043-X

[17] R. J. Moffat, “Experimental Heat Transfer,” Proceedings of 9th International Heat Transfer Conference, Jerusalem, Vol. 1, 1991, pp. 308-310.

[18] T. V. Jones, Z. Wang and P. T. Ireland, "The Use of Liquid Crystals in Aerodynamic and Heat Transfer Experiments," Proceedings of 1st ImechE Seminar on Optical Methods and Data Processing in Heat and Fluid Flow, 
London, 1992, pp. 51-65.

[19] S. Ashforth-Frost, L. S. Wang, K. Jambunathan, D. P. Graham and J. M. Rhine, "Application of Image Processing to Liquid Crystal Thermography,” Proceedings of 1st ImechE Seminar on Optical Methods and Data Processing in Heat and Fluid Flow, London, 1992, pp. 121-126.

[20] W. Leiner, K. Schulz, M. Behle and S. Lorenz, "Imaging Techniques to Measure Local Heat and Mass Transfer," Proceedings of $3^{\text {rd }}$ IMechE Seminar Optical Methods and Data Processing in Heat and Fluid Flow, London, 1996, pp. 1-13.

[21] T. A. Kowalewski, P. Ligrani, A. Dreizler, C. Schulz and U. Fey, "Temperature and Heat Flux: Thermochromic Liquid Crystals,” In: C. Tropea, A. L. Yarin and J. F. Foss, Eds., Springer Handbook of Experimental Fluid Mechanics, Springer, Berlin, 2007, pp. 487-500. http://dx.doi.org/10.1007/978-3-540-30299-5_7

[22] A. Jung and J. Zuber, "Thermographic Metohds in Medical Diagnostics,” Medpress, Warsaw, 1998 (in Polish).

[23] S. Klosowicz, E. Nowinowski-Kruszelnicki and J. Zmija, "The Simple Method to Prepare Polymer Dispersed Liquid Crystals,” Molecular Crystals and Liquid Crystals, Vol. 215, No. 1, 1992, pp. 253-255 (in Polish). http://dx.doi.org/10.1080/10587259208038532

[24] J. Zmija, S. Klosowicz and S. Borys, "Cholesteric Liquid Crystals in a Detection of Radiation," WNT, Warsaw, 1989 (in Polish).

[25] MINCO Products, Inc., Minnesota, USA, 2006.

[26] MERCK Ltd., “Thermochromic Liquid Crystals,” Poole, 2006.

[27] D. Mikielewicz, A. Stasiek, M. Jewartowski and J. Stasiek, "Measurements of Heat Transfer Enhanced by the Use of Transverse Vortex Generators," Applied Thermal Engineering, Vol. 49, 2012, pp. 61-72. http://dx.doi.org/10.1016/j.applthermaleng.2011.11.013

[28] G. Tanda, "Heat Transfer, Pressure, Drop in a Rectangular Channel with Diamond-Shaped Elements," International Journal of Heat and Mass Transfer, Vol. 44, No. 18, 2001, pp. 3529-3541. http://dx.doi.org/10.1016/S0017-9310(01)00018-7

[29] G. Tanda, "Heat Transfer in Rectangular Channels with Transverse and V-Shaped Broken Ribs," International Journal of Heat and Mass Transfer, Vol. 47, No. 2, 2004, pp. 229-243.

http://dx.doi.org/10.1016/S0017-9310(03)00414-9 